\title{
Cambios y continuidades en el Levante (ca. 1300-900 a.n.e.): Una propuesta de síntesis sociopolítica
}

\section{(1) Emanuel Pfoh \\ Consejo Nacional de Investigaciones Científicas y Técnicas / Universidad Nacional de La Plata, Argentina}

Fecha de recepción: 15 de agosto de 2019. Fecha de aceptación: 14 de septiembre de 2019.

\section{Resumen}

Entre 1300 y 900 a.n.e., las regiones de Siria y de Palestina experimentaron una serie de disrupciones y reacomodamientos, especialmente en la escena política. Más aún, la opinión historiográfica tradicionalmente aceptada indica que un cambio radical en las estructuras sociopolíticas ocurrió, marcando una transición de organizaciones políticas territoriales a organizaciones "nacionales" o étnicas. La discusión presentada en este trabajo apunta a revisar dicha opinión y a observar una permanencia fundamental, luego de la crisis del siglo XII, de las estructuras jerárquicas basadas en el parentesco y el patronazgo; estructuras sociopolíticas que proporcionaban las pautas básicas en la dinámica interna y externa de los reinos locales. Finalmente, se presenta una hipótesis al respecto de la relevancia de la adscripción y la subordinación políticas en la creación de identidades étnicas en el Levante durante la Edad del Hierro.

\section{Changes and Continuities in the Levant (ca. 1300-900 BCE): A Socio-Political Synthesis}

\footnotetext{
Abstract

Between 1300 and 900 BCE the regions of Syria and Palestine experienced a series of disruptions and rearrangements, especially in the political scene. Further, the traditionally accepted historiographical view indicates that a radical

"Una primera versión de este artículo apareció publicada en inglés (Pfoh, 2018). La presente versión en español ha sido ligeramente revisada, actualizada y ampliada. Agradezco a la Agencia Nacional de Promoción Científica y Tecnológica por financiar el proyecto PICT 2016-1269, el cual dirijo, que hizo posible esta investigación.
}

Palabras clave

Siria-Palestina Edad del Bronce Tardío Edad del Hierro sociopolítica patronazgo etnicidad

Keywords

Syria-Palestine Late Bronze Age Iron Age Socio-Politics Patronage Ethnicity 
Véase, por ejemplo, Albright (1939). Véase también la crítica en Lemche (1985: 48-62, 148-152); también Silberman (1998); MasettiRouault (2009; [en prensa]). Una reciente opinión sobre la identidad étnica y las migraciones durante el siglo XII a.n.e. se encuentra en Niesiołowski-Spanò (2016a: 1-11). change in socio-political structures occurred, marking a transition from territorial polities to "national" or ethnic polities. The discussion offered in this paper aims at challenging such a view and observing the fundamental permanence, after the twelfth century crisis, of hierarchical structures based on kinship and patronage; socio-political structures which informed the basic internal and external dynamics of the local kingdoms. Lastly, it is presented a hypothesis concerning the relevance of political attachment and subordination for the creation of ethnic identities in the Iron Age Levant.

Los cambios y las continuidades sociopolíticos han sido, durante mucho tiempo en la historiografía, herramientas conceptuales de explicación y de comprensión de diversas realidades históricas. En efecto, la idea de cambio histórico, aparte de las concepciones escatológicas del tiempo, puede ser rastreada -por ejemplo, y desde una concepción eurocéntrica de la historia- hasta las propias interpretaciones contemporáneas de la Revolución Francesa de 1789, pero también como un elemento clave en el pensamiento progresista y evolucionista del siglo XIX, notablemente con la concepción darwinista en biología y la visión marxista de la historia. No obstante, bien podría afirmarse que es recién durante las décadas de 1960 y 1970, a partir de los intensos debates planteados en el seno de las ciencias sociales y humanas, en especial sobre la naturaleza misma de la historia, que este par analítico -junto con la identificación conceptual tanto de regularidades como de singularidades en la vida social a través del tiempoalcanzó una aceptación generalizada como elemento constitutivo esencial de la comprensión histórica sobre los diferentes aspectos y variaciones del pasado humano (véase, por ejemplo, Gaddis, 2002; Fritzsche, 2004; Megill, 2007).

Las historiografías tradicionales sobre el Cercano Oriente antiguo y los escenarios bíblicos durante el siglo XX postularon la transición de la Edad del Bronce Tardío a la Edad del Hierro en el Levante (en torno a 1200 a.n.e.) a partir de la evocación de un cambio estructural producido por poblaciones invasoras (semi-)nómades, una concepción probablemente deudora de la imagen bíblica de los israelitas conquistando la tierra de Canaán, como se narra en el libro de Josué, en conjunto con las referencias egipcias sobre el arribo de los Pueblos del Mar. No obstante, no puede descartarse, junto con estas imágenes, la duradera recepción en Europa de los siglos XVIII y XIX de noticias sobre pueblos germánicos penetrando la periferia del imperium romano, para luego instalarse en el territorio, combatir las poblaciones locales y eventualmente desplazarlas o, en ciertos casos, mezclarse con ellas, durante las primeras centurias de nuestra era. ${ }^{1}$

Tales imágenes de procesos rápidos y casi absolutos de reemplazos étnicos han sido recientemente revisadas en varios campos de la historiografía (p. ej., Geary, 2002; también Wiedemann, Hofmann y Gehrke, 2017). En los estudios relativos a las realidades antiguas del Levante, esta revisión también tuvo lugar, en parte gracias al progreso en las investigaciones arqueológicas de la región, pero fundamentalmente a partir de un reajuste en los paradigmas interpretativos del cambio social e histórico (Lemche, 1985; Coote y Whitelam, 1987; Finkelstein, 1988; Thompson, 1992; Finkelstein y Na'aman, 1994; Grabbe, 2008; 2010; Killebrew y Lehmann, 2013). A continuación, abordaremos sintéticamente 
estas cuestiones en tres secciones, tratando (1) los factores generales de la transición del siglo XII en lo que respecta a cuestiones de sociopolítica; ${ }^{2}$ (2) los cambios sociopolíticos; y (3) las continuidades sociopolíticas. Finalmente, concluimos con comentarios sobre la cuestión de la identidad étnica en estas comunidades políticas, un tema aún abierto y en actual reconsideración, que requiere de mayor discusión y elaboración interpretativa.

\section{La transición de la Edad del Bronce Tardío a la Edad del Hierro}

En la reciente historiografía del Cercano Oriente antiguo, la transición del siglo XII es concebida como una situación crítica en la que una serie de cambios estructurales, especialmente en la región del Mediterráneo oriental, ocurrieron: la desaparición del comercio de larga distancia, que había caracterizado a la Edad del Bronce, junto con una economía centrada en el palacio; el reemplazo de estados territoriales por estados étnicos, lo que implica la aparición en la escena política e identitaria de "nuevos pueblos", provenientes de allende el mar y del desierto; la aparición de nuevas tecnologías (la difusión de la metalurgia del hierro, el uso amplio de camélidos por parte de comerciantes de larga distancia, la difusión de la escritura alfabética; Ward y Joukowsky, 1992; Gitin, Mazar y Stern, 1998; Liverani, 2014: 381-400; Cline, 2014).

Desde la perspectiva de la organización sociopolítica, el cambio más significativo en la escena regional pareciera haber sido el reemplazo de una topografía política fragmentada durante la Edad del Bronce Tardío (ca. 1550-1200 a.n.e.) tanto en Siria como en Palestina, algo especialmente visible en la correspondencia de El Amarna de la segunda mitad del siglo XIV, por el surgimiento de comunidades políticas de relativa homogeneidad y extensión territorial desde inicios de la Edad del Hierro II (ca. 1000-600 a.n.e.) en adelante: los reinos neo-hititas y arameos en Siria; las "ciudades-estado" filisteas y fenicias en la costa de Palestina y los reinos de Israel y de Judá en las tierras altas; y en Transjordania, los reinos de Ammón, Moab y Edom (Pfoh, 2013). Más aún, la comprensión de una transición sociopolítica de pequeñas ciudades-estado territoriales durante el Bronce Tardío a estados étnicos/"nacionales" durante la Edad del Hierro estuvo beneficiada de un consenso académico en los estudios del Cercano Oriente antiguo desde la publicación de la influyente obra de Giorgio Buccellati Cities and Nations of Ancient Syria en $1967 .{ }^{3}$ La percepción de Buccellati de una organización esencialmente territorial de las polities de la Edad del Bronce dependía en última instancia de una lectura de la correspondencia diplomática de las cartas de Alalab̧, Ugarit, Mari y del archivo de El Amarna, la cual exponía una ordenación política compuesta de pequeños reyes que eran señores de sus súbditos y "vasallos" de un rey externo y de mayor jerarquía (Buccellati, 1967: 25-74). Desde esta perspectiva, y al igual pues que en tiempos medievales en Europa, los reinos de la Edad del Bronce, tanto las ciudades-estado como las polities de mayor alcance regional, eran organizaciones territoriales en su estructuración pero también en su concepción y terminología política. El comienzo de la Edad del Hierro marcó, de acuerdo con Buccellati, la aparición de organizaciones tribales y "gentilicias" provenientes de las zonas desérticas o periféricas a las zonas urbanas y su elevación a la
2. Con "sociopolítica" referimos, básicamente, a las prácticas de una particular organización política que también comprenden una ontología política, configuradas todas ellas por características culturales, históricas y geográficas, y que además pueden ser analizadas a partir de una analogía comparativa de ejemplos y situaciones etnográficos.
3. Buccellati no ha cambiado su interpretación básica en su más reciente ensayo Alle origini della politica (Buccellati, 2013: 257-261). 
4. La comprensión que exponemos aquí de la noción de "organización tribal/tribu" denota una comunidad política en la que la dinámica de parentesco y la ideología de una descendencia de un ancestro común sostiene una correspondiente política tribal. En contraste, una comunidad política estatal, si bien admite la existencia en su interior de organizaciones tribales, oblitera la hegemonía de la política tribal bajo la propia política estatal (vale decir, el monopolio legítimo de la coerción). Véase, por ejemplo, la presentación en Tibi (1990) para el moderno Medio Oriente. 5. Véase también Liverani (2002: 38-41).

6. Finkelstein (2003: 81 [los primeros dos énfasis son originales; el último es nuestro]). Véase también Finkelstein (2013). Desde la misma perspectiva, Sergi (2015) define al reino de Judá como territorial pero también (junto con Moab) como "nacional". hegemonía política regional, eventualmente manifestada en la forma de estados nacionales, como se atestiguaría especialmente en el Antiguo Testamento con el ejemplo de Israel (Buccellati, 1967: 75-135). ${ }^{4}$

Como hemos observado ya, esta concepción de transición sociopolítica ha sido dominante en la historiografía cercano-oriental durante las últimas décadas. Al respecto, por ejemplo, Mario Liverani (1997: 114) observaba:

In the old system (the Bronze Age system) states were based on territory: everybody residing inside a given territory was the subject of the local king. The dominance of one polity over another was therefore simply depending on their respective forces. With the coming of the Iron age, a new factor became relevant: ethnicity. Inside a given polity, ethnicity provided a much more effective tool for solidarity, compaction, and identification. ${ }^{5}$

Israel Finkelstein, también, ha ofrecido un escenario de transición algo más complejo, no tan mecánico, como el presentado por Buccellati, para la aparición de nuevas comunidades políticas a comienzos del Hierro en las tierras altas de Canaán, aunque reteniendo algo de la concepción pionera de Buccellati:

The Omride state therefore also demonstrates both change and continuity. There was change in the sense that the Omrides succeeded in creating a territorial state covering large areas of both highlands and lowlands. There was continuity in the sense that this was an age-old dream of hill country rulers. Indeed, $9^{\text {th }}$-century Samaria was not very different, conceptually or architecturally, from Labayu's Shechem.

The real revolution in political dynamics came only in the $8^{\text {th }}$ century, with the integration of the Northern Kingdom into the Assyrian economic hegemony and the rise of $a$ true national state in Judah. ${ }^{6}$

Luego de cinco décadas de que esta concepción se haya mantenido como el marco interpretativo mayormente aceptado para la sociopolítica del Levante, ambas categorías -estado territorial y estado étnico/nacional- deben ser reconsideradas en su utilidad interpretativa. A continuación, proponemos distinguir dos aspectos analíticos para abordar esta cuestión: la organización política (la estructura sobre la cual la política está basada y se conduce) y la práctica política (la dinámica y la expresión concreta de la política, las cuales a su vez reproducen la organización sociopolítica). Esta distinción permite reacomodar de mejor manera nuestro conocimiento sobre el cambio y la continuidad en la transición del Bronce Tardío al Hierro.

\section{Cambios sociopolíticos}

\subsection{Organización política}

Las comunidades sociopolíticas de Siria-Palestina durante la Edad del Bronce Tardío eran estructural y espacialmente limitadas en su tamaño y se encontraban en un escenario político constantemente fragmentado (Savage y Falconer, 2003; 
Jasmin, 2006a; Benz, 2016). ${ }^{7}$ En Siria, hallamos un desarrollo urbano relativamente mayor con respecto a Palestina, posiblemente debido a la ventaja geográfica que permitió una temprana influencia desde el norte de Mesopotamia (aunque esta cuestión es aún un tema en discusión; véase Akkermans y Schwartz, 2003: 211-287). Sin embargo, tal constatación de urbanismo no debería conducirnos, necesariamente, a prescribir la presencia de organizaciones sociopolíticas de una "complejidad estatal" en la zona, tales como las halladas en Egipto y en la Baja Mesopotamia, si bien en una gradación menor de evolución social. ${ }^{8}$ En Palestina, muy probablemente a causa de la baja densidad demográfica durante este período (cf. Bunimovitz, 1994), pero también a la alta probabilidad de un predominio de estructuras de parentesco en la organización general de la población rural y pastoralista en asociación con actores sedentarizados (Lehmann y Niemann, 2006), se podría pensar para estos siglos la existencia de una organización sociopolítica local anclada tanto en el parentesco básico y el orden "tribal" como en estructuras afines al parentesco extendido en el paisaje político circundante (como el patronazgo y las redes de clientela, estructurados en un orden patrimonial), en las que el monarca de la comunidad política era en realidad más un hombre de prestigio que de poder concreto en la sociedad, una condición, en consecuencia, difícilmente relacionada a cualquier tipo de organización estatal, no obstante la presencia de escribas y de comunicación diplomática en las cortes de los pequeños reyes del Levante (cf. Pfoh, 2016: 98-105, 159-160; 2019).

Durante la Edad del Hierro I (ca. 1200-1000 a.n.e.), la preponderancia de lo que ha sido referido como un elemento tribal en la organización social del Levante debería más bien ser comprendida como un reflejo de la permanente fragmentación sociopolítica de la región y una supervivencia de parte del sistema social dislocado a fines del Bronce Tardío en Canaán, ahora expandido en su potencial al carecer de un límite político externo (véase ya Alt, 1959b). No podemos incluir aquí a la Monarquía Unida de David y Salomón como evento sociopolítico en la escena política del Levante a comienzos de la Edad del Hierro puesto que la existencia de dicha organización es más bien bíblica (i.e., mítica) que propiamente histórica (véase, por ejemplo, la evaluación en Finkelstein, 2010). A partir del siglo $X$ a.n.e., los reinos arameos de Siria manifiestan este elemento tribal en su nominación política, como Bìt Agusi, Bìt Adini, etc., indicando una ordenación articulada a partir de la adscripción a una "casa de NP". ${ }^{9}$ El reino de Israel en el siglo IX es también conocido en las fuentes neoasirias bajo esta nominación, vale decir, como Bìt Humri (Hallo, 2003: 267268; también Kelle, 2002). En Transjordania, las respectivas comunidades políticas de Ammón, Moab y Edom han sido categorizadas asimismo como "reinos tribales" o, inclusive, "estados tribales", a causa de su organización territorial y genealógica, oscureciendo en gran medida los aspectos más relevantes de una pretendida organización estatal. ${ }^{10}$ Sin embargo, dentro de su heterogeneidad, dos factores parecerían ser novedosos en la configuración sociopolítica de todas estas organizaciones, en mayor o menor medida: la condición de estatalidad y la presencia de una identidad étnica. A menudo en la historiografía del Levante en la Edad del Hierro, estas polities suelen ser comprendidas como "estados secundarios", cada una de ellas con un correspondiente trasfondo étnico mayormente claro y específico, vinculado a sus específicas afiliaciones tribales (Knauf, 1992; LaBianca y Younker, 1995; Joffe, 2002). Las “ciudades-estado" filisteas y fenicias representarían, por su parte, un nuevo fenómeno sociopolítico en el
7. Este orden sociopolítico ya había sido reconocido por Alt (1959a: 67), notando una continuidad en los sitios de autoridad política ("Herrensitze", que en rigor tienen la connotación patrimonial de household) mencionados en los Textos de Execración (ca. 1800 a.n.e.) y en las cartas de El Amarna.

8. Acerca de la noción de urbanismo, Schloen (2016: 447) observa: "Archaeologists rather casually describe any walled settlement as 'urban' and refer to an increase in the number of walled settlements as 'urbanization.' But these terms carry theoretical baggage that is not appropriate for the case at hand [Israel y Judá en la Edad del Hierro]. The term 'urban' implies far more than a purely descriptive distinction between walled and unwalled settlements. It is not an architectural term but a sociological term that implies an economic contrast between specialized cities and unspecialized villages which are the locus of staple production but are dependent on urban centers for manufactured goods and professional services, creating an economic dynamic that was famously described (and wrongly assumed to be universal) by Adam Smith in his Wealth of Nations".

9. "The polities constituted at this time by the Arameans-which may be singled out by the distinctive onomastics of the local rulers or chiefs-often betray by their very toponymy (formed along the pattern Bìt + PN, with PN indicating an eponymous ancestor) an origin in a sphere of kinship and tribal/clanic solidarity of long standing." (Fales, 2017: 153). Véase también Leonard-Fleckman (2018).

10. Routledge (2004) ha propuesto aplicar el concepto de segmentary state, más conocido en los estudios africanistas modernos (cf., i.e., Southall, 1988), en reemplazo de la noción de "estado tribal". La cuestión problemática con esta terminología reside, en nuestra opinión, no tanto en la adjetivación sino en el propio sustantivo empleado, en términos de conceptos analíticos. 
11. Niemeyer (2000), se refiere, aunque algo vagamente en términos de análisis tipológico, a las polities fenicias en torno a 1000 a.n.e. como "city-kingdoms or city principalities" (p. 91), conceptos algo más apropiados que la noción de "ciudad-estado", que ciertamente no hace justicia a la configuración de los asentamientos urbanos del Levante que actuaban como centros políticos. De igual modo, Strange (2000: 135-136) se refiere en general a las ciudadesestado filisteas como parte de una confederación, cada una de ellas gobernada por reyes (los seranim bíblicos), aunque sin ninguna indagación exhaustiva sobre su configuración sociopolítica.
Levante meridional durante la Edad del Hierro; con todo, su organización sociopolítica se asemeja en mucho, en verdad, a las "ciudades-estado" de la Edad del Bronce Tardío (Belmonte, 2003; Yasur-Landau, 2010; NiesiołowskiSpanò, 2016a: 144-179). ${ }^{11}$

\subsection{Práctica política}

Las cartas de El Amarna, en particular, nos habilitan una representación bastante acabada de la dinámica de las pequeñas polities locales en Siria-Palestina, a la sombra del dominio territorial de los grandes poderes de la segunda mitad del segundo milenio a.n.e.: Hatti y, por cierto tiempo, Mittani en Siria; Egipto en Siria meridional y Palestina. En la escena política local, alianzas entre las pequeñas comunidades políticas -en rigor, alianzas personales entre los respectivos monarcas-, así como el conflicto y la competencia entre pares líderes, marcaban el ritmo de la práctica sociopolítica, no sólo durante el siglo XIV sino, muy probablemente, durante toda la Edad del Bronce.

En la escena regional, los grandes reyes gobernaban la región de dos modos distintivos, en general: el reino de Hatti gobernaba sobre los pequeños reinos sirios a través de tratados de subordinación impuestos a los monarcas locales; Egipto, en cambio, había emplazado guarniciones y centros administrativos con sus comisionados en Palestina para mantener el control y la inteligencia militar sobre los pequeños reyes locales y garantizar el envío de tributo y la circulación de bienes primarios, así como de sus tropas. Posteriormente, durante el siglo XIII a.n.e., la instalación de "residencias de gobernador" como centros de operaciones políticas locales, reajustaría el control egipcio en el Levante meridional por un par de siglos más, aunque de manera decreciente en su capacidad de ejecución, vinculado a las vicisitudes internas y externas de Egipto (Oren, 1984; también, más recientemente, Koch, 2018; Morris, 2018: 117-186, 192-217).

La crisis del siglo XII desmanteló primeramente el dominio hitita sobre el Levante septentrional y posteriormente gran parte del egipcio en la zona meridional de la región. En los siglos posteriores inmediatos, como ya observamos, la ausencia de un poder regional permitió que las organizaciones sociopolíticas locales -articuladas en relaciones de parentesco y de patronazgo, y con una organización general de corte tribal- se desarrollaran y expandieran para el siglo IX en comunidades políticas de variada extensión territorial, con la capacidad de conquistarse las unas a las otras -Israel sobre Moab (Vera Chamaza, 2005: 29-60); Aram sobre Israel (Kleiman, 2016)aunque solamente por un breve período de tiempo. Todos estos reinos, eventualmente, se incorporaron a la órbita asiria, en expansión desde el norte de Mesopotamia hacia el Levante, ya sea indirectamente, como reinos "vasallos", o formalmente como nuevas provincias asirias; una estructura de subordinación que posteriormente pasaría a manos del dominio neobabilónico y persa (cf. Knauf y Guillaume, 2016: 85-133; también Vera Chamaza, 2005: 61-119; Lipschits, 2004: 37-52). 


\section{Continuidades sociopolíticas}

\subsection{Organización política}

Los patrones de asentamiento, tanto en Siria como en Palestina y salvo menores excepciones, manifiestan una continuidad evidente de la Edad del Bronce Tardío a la del Hierro, con una importante expansión demográfica hacia las tierras altas de Palestina durante el Hierro I (Whitelam, 2002; Dornemann, 2003; Finkelstein, 2003: 76-78; Jasmin, 2006b; Sader, 2016; para Filistea, cf. Yasur-Landau, 2010: 282-300, 340-342). Los centros sociopolíticos del Bronce Tardío reemergen en el Hierro I, y debería esperarse -sin que esto sea en realidad sorprendente- la reaparición de similares patrones de organización sociopolítica (i.e., Shechem y Jerusalén durante el Bronce Tardío; Samaria y Jerusalén durante el Hierro II; Finkelstein y Na'aman, 2005). Una diferencia clave, con respecto al período anterior, es la ausencia durante el Hierro I e inicios del Hierro II de poderes regionales externos en el Levante, lo cual permitió el desarrollo de comunidades políticas autóctonas, como la dinastía omrita centrada en Samaria, a la que aludía Finkelstein más arriba. No obstante, si tomamos en cuenta la continuidad en los asentamientos así como el nucleamiento demográfico y la expansión territorial de los principales sitios de Palestina durante el Hierro I, tal vez debería pensarse mejor en un desarrollo cuantitativo de elementos y características ya existentes en el período anterior y ahora carentes de un condicionante externo en su dinámica sociopolítica, antes que pensar en un salto político cualitativo en la organización de la sociedad, vale decir, un cambio evolucionario de una sociedad de jefatura a una sociedad estatal, no obstante la presencia de algunas características propias de lo estatal en la región durante el siglo IX. ${ }^{12}$

\subsection{Práctica política}

Podría afirmarse que la historiografía sobre el Cercano Oriente antiguo y sobre la Biblia hebrea ha considerado siempre a la práctica de la subordinación política de los pequeños reinos del Levante en términos de "vasallaje", con una consecuente dinámica política entre señor y sirviente, aunque sin haber discutido o problematizado dicha conceptualización o haber justificado el empleo de la terminología. En otra contribución, hemos argumentado en contra del uso de estos términos propios de las relaciones feudovasalláticas de la Europa medieval y a favor del concepto de patronazgo, o de relaciones patrón-cliente, como un mejor modo de comprender la dinámica de la subordinación ante poderes externos en el Levante (Pfoh, en prensa). Si analizamos, por ejemplo, los modos en que los pequeños reyes siro-palestinos expresaban su lealtad política a sus amos externos, no resulta en absoluto difícil percibir indicios claros de una dinámica de patronazgo: la lealtad política es siempre personal y se encuentra atada a una expectativa de reciprocidad, aun cuando ésta suele manifestarse de manera asimétrica y condicionada por la parte superior de la alianza política; precisamente, el lazo político entre un gran rey y un pequeño rey es siempre impuesto por el primero, junto con condiciones que siempre le son favorables, y se encuentra sujeto a una disolución situacional e imprevista (en rigor, los tratados entre dos reyes no poseen las mismas expectativas institucionales de los pactos de vasallaje medievales).

12. A favor de percibir estatalidad en el Levante meridional durante la Edad del Hierro, cf. de manera representativa Dever (1994; 1997); Holladay (1995); SchäferLichtenberger (1996); Na'aman (2007); Garfinkel et al. (2012). Para una crítica de modelos y teorías del surgimiento del estado, usuales en la historiografía del "antiguo Israel”, cf. Pfoh (2008). Para otras problematizaciones sobre el Levante meridional durante la Edad del Hierro, cf. Kletter (2004: 14-29); Tebes (2016); Routledge (2017: 59-67). 
13. Véanse por ejemplo $E A 77,85$, 117, 118, y 130. La más reciente traducción (y transliteración) de las cartas de El Amarna se encuentra en Rainey (2015).

14. Niemann (2008); Nam (2012). Cuando referimos aquí a una sociedad de jefatura, no lo hacemos a través de un modelo neoevolucionista, a través del cual el Estado tiene un advenimiento necesario e inevitable (como lo expresa, por ejemplo, Dever, 1994), sino como un concepto analítico que permite ordenar la información empírica, textual y arqueológica, de que disponemos; cf. Pfoh (2008).
Otro indicio de importancia que apunta a una dinámica política de patronazgo en la región reside en el modo en que los monarcas locales construyen su autoridad hacia el interior de su reino: inevitablemente, deben responder antes las demandas y reclamos de sus súbditos; los pequeños reyes deben proveer -como buenos patrones- para ser obedecidos y seguidos lealmente. Durante la Edad del Bronce Tardío, hallamos evidencia de estas situaciones en las cartas de El Amarna. ${ }^{13}$ Pero también luego, durante la Edad del Hierro II en Palestina, la información provista por los óstraca de Samaria podría interpretarse a través de un prisma de patronazgo, según el cual los intercambios de bienes primarios (vino y aceite) entre la corte real en Samaria y los clanes de su periferia, realizados entre individuos con nombres propios en la evidencia (no con títulos administrativos o cargos de funcionarios reales), nos indican un proceso de construcción de autoridad monárquica en lo que debería comprenderse primero como una sociedad de jefatura antes que un estado incipiente. ${ }^{14}$ Estos indicios, asimismo, nos podrían estar indicando también una pervivencia, luego de la crisis del siglo XII, de la práctica del patronazgo, siendo éste, en efecto, el principal vehículo de expresión política e ideológica de los monarcas locales de Siria-Palestina.

\section{La sociopolítica en la larga duración y la ilusión étnica}

Como mencionábamos al comienzo, las actuales investigaciones sobre la transición sociopolítica desde la Edad del Bronce Tardío a la Edad del Hierro aún se encuentran, en mayor o menor medida, bajo la influencia del paradigma de las invasiones bárbaras: una generación atrás, arameos provenientes del desierto e israelitas que se sedentarizaban luego de transitar como semi-nómades las estepas áridas del Levante constituían el principal factor en la aparición de nuevas realidades sociopolíticas y étnicas en la región. En la actualidad, el impacto sociopolítico de estos nuevos actores históricos a inicios del Hierro se ha reducido considerablemente: una conquista militar israelita se encentra descartada en términos históricos, la realidad histórica de una Monarquía Unida israelita ha sido profundamente debatida y la posibilidad de encontrar trazos confiables en el registro arqueológico de identidades étnicas resulta incierta y discutible. Sin embargo, la noción de que la aparición, como novedad sociopolítica nativa, de estructuras estatales de algún tipo pueden ser identificadas en el registro arqueológico del Levante durante este período, vinculadas en gran medida con la aparición en el registro epigráfico (y textual, si atendemos a las noticias que presenta el Antiguo Testamento) de nuevas realidades étnicas en la región, permanece como algo mayormente indiscutido y tácitamente aceptado, no obstante el hecho de que una simple comparación entre los sitios fortificados de la Edad del Bronce Medio II (ca. 2000-1550 a.n.e.) y del Hierro II hallaría construcciones y edificaciones mucho más monumentales en el primer período, en donde los arqueólogos no hallan estados territoriales propiamente (pace Buccellati) sino ciudades-estados en las que la estatalidad no se ha manifestado plenamente. No obstante la medida cuantitativa de este diacrítico monumental, debe observarse que la monumentalidad por sí misma no es un factor obvio e innegable de un aparato estatal detrás de su producción (cf. Osborne, 2014: 8-9). 
La expansión territorial de las polities del Hierro II es también algo percibido usualmente como una característica de estatalidad (en conjunto, especialmente en Judá durante el siglo VII, con la presencia de edificios públicos, fortificaciones fronterizas, escritura administrativa, etc.; Faust, 2012). No obstante, la dinámica sociopolítica de los reinos arameos en Siria y del reino de Israel en Palestina durante los siglos IX y VIII bien podría ser comprendida como esencialmente tribal en la ingeniería de sus alianzas y modos políticos de subordinación y dependencia personales, de manera análoga a lo que sucedía durante el Bronce Tardío, aunque sin la presencia de grandes poderes externos dominando la región y supervisando los contactos políticos. A pesar de la usual referencia a una presencia de características estatales en los distintos reinos del Levante (tanto en los mal llamados "nacionales" como en aquellos definidos como "estados tribales"), las dinámicas de la política y de la expansión y control territoriales se encuentran conducidas no por una lógica propiamente estatal, a través de una administración burocrática y un ejército profesional, sino más bien de acuerdo con una lógica que bien podría corresponderse con una organización propia de una jefatura compleja: los monarcas levantinos de los siglos IX-VIII, al igual que los monarcas levantinos del período de El Amarna, son -de nuevo- más bien jefes tribales que propios líderes estatales, siendo la principal diferencia entre ambos períodos la extensión del control territorial: limitado a la casa, palacio o residencia del monarca y su periferia inmediata durante el Bronce Tardío y mucho más expandido durante el Hierro II, pudiendo abarcar otros reinos o polities bajo sujeción. Estos monarcas levantinos, en especial durante los siglos IX y VIII a.n.e., no gobernaban sobre una sociedad estatal sino sobre una sociedad esencialmente tribal, en estructura y dinámica, en la que ellos eran los líderes militares, protegiendo a la comunidad de las amenazas externas, conduciéndola en batalla e intermediando con poderes superiores. Así pues, y desde una perspectiva de larga duración, la historia sociopolítica de Siria-Palestina refleja en efecto una serie de eventos políticos y cambios aparentes, pero estructuras más profundas, como el parentesco y la práctica del patronazgo, resultan resilientes a estos cambios superficiales, y ellas reaparecen luego de las crisis ocurridas - como la del siglo XII- readaptándose bajo nuevas situaciones sociales e históricas. ${ }^{15}$

Recientemente, dos investigadores han afirmado que "the idea of the sovereignty of small states in the area [el Levante] is an illusion based on temporary neglect by imperial powers" (Knauf y Guillaume, 2016: 145). Uno podría preguntarse, entonces, hasta qué punto puede existir un estado sin soberanía propia sobre su territorio y considerar, nuevamente, si los monarcas levantinos, tanto durante el Bronce Tardío como en el Hierro, poseían más bien autoridad y prestigio, antes que un poder político centralizado e institucionalizado, en sus respectivas comunidades, tal como lo hace un jefe tribal, y que debían mantener ese orden sociopolítico a través de intercambios personalizados. ${ }^{16}$ Luego de pensar la cuestión por fuera del marco interpretativo del estado (cf. Smith, 2003), para organizar el análisis de la información arqueológica y epigráfico-textual en las Edades del Bronce Tardío y del Hierro en el Levante, y atendiendo también a las prácticas políticas constatadas, sería posible entonces argumentar que graduaciones y variaciones de relaciones de patronazgo - desde la comunidad local a las relaciones locales inter-polity
15. Podrían, entonces, revisarse las conclusiones, aunque no tanto así el análisis propio, del de otro modo excelente estudio de Finkelstein (1994). Cf. también Whitelam (2002: 400-401).

16. Pfoh (2013); cf. también, comparativamente, el análisis en Cooper (2010) sobre la hegemonía de Ebla sobre su periferia. 
17. Lemche (2002: 265) Ilama a este tipo de comunidad política patronage state: "By "patronage state' I mean a state that is organized around a ruling family as its personal possession. It does not include any extended kind of bureaucracy except for what is necessary to regulate the relations between the ruling family-the patrons-and its clients, the ordinary population. That kind of state dominated the area during the Late Bronze Age, and it did as a matter of fact survive as a typical form of political organization well into the Iron Age". En otra parte, hemos argumentado a favor de concebir esta organización sociopolítica, antes que como "Estado + adjetivo", como un patronage kingdom, un reino articulado mayormente a través de redes de patronazgo (Pfoh, 2008: 111), acordando implícitamente con Kletter (2004: 28) en la preeminencia conceptual del "reino" para interpretar la sociopolítica del Levante.

18. Sumamente pertinentes para cuestionar la identificación de cultura material "israelita" en las tierras altas de Palestina durante

el Hierro I son los estudios de London (1989); Skjeggestad (1992); y Zwingenberger (2001). No obstante estas críticas metodológicas, en la arqueología ("bíblica") de Palestina existe una tendencia generalizada a identificar grupos étnicos en el registro arqueológico;

cf. por ejemplo Killebrew (2005).

19. Si tal expresión resulta de alguna efectividad analítica: para un argumento en contra de su uso, cf. Porzia (2018).

20. Para una perspectiva "proétnica”, véase Dever (2007); pero compárese con Kletter (2014). De hecho, como Jasmin (2006b: 202) observa: "En particulier, l'étude de diverses catégories céramiques dans le sud de la Palestine au cours du Fer I indique plutôt des frontières géopolitiques et culturelles marquées que des frontières strictement ethniques". Véase más sobre la relación entre etnicidad y arqueología en Jones (1997).

21. Para información etnográfica que demuestra la relación conceptual y analítica entre patronazgo y etnicidad, véase por ejemplo Barth (1969; 1981); Lemarchand (1972); Berman (1998); Berman, Cottrell y Ghai (2009).

Para el carácter situacional de ciertas identidades étnicas, cf. orientativamente Okamura (1981). a la subordinación local ante poderes foráneos- marcan la configuración de la política en ambos períodos. Desde esta perspectiva, luego de la crisis del siglo XII, ocurrió un reacomodamiento de estructuras de patronazgo a través de las nuevas comunidades políticas que aparecieron, permitiendo su expansión territorial como modo de relación política en la región, ${ }^{17}$ hasta el advenimiento de la conquista asiria del Levante durante el siglo VIII a.n.e., la cual igualmente empleó este tipo de vinculación sociopolítica para ejercer la subordinación de las polities conquistadas (cf. Berlejung, 2012; Bagg, 2013; Baruchi-Unna, 2018; también Pfoh, 2013: 33-35).

Con respecto, finalmente, a la cuestión de la etnicidad durante la Edad del Hierro y a su inscripción sociopolítica dentro de los nuevos "estados étnicos/ nacionales" del período, también podemos avanzar una revisión. Recientes investigaciones sobre la cuestión de la etnicidad en el Levante, y en particular en el Levante meridional, no han arrojado conclusiones definitivas acerca de aquello que el registro arqueológico nos informa a partir de un análisis de la cultura material y los patrones de asentamiento. En efecto, la ambigüedad del material arqueológico, visto a través de elaboraciones etnográficas y etnohistóricas, apunta a una identificación no conclusiva de concretas identidades étnicas (con la posible excepción de repertorios cerámicos claramente filisteos o propios de los Pueblos del Mar). ${ }^{18}$ Con certeza, solamente podemos asumir la presencia de diferentes expresiones culturales y políticas locales, pero ello no es suficiente para esclarecer el "mosaico étnico" ${ }^{19}$ del Levante en este período, o en otras palabras, al no contar con testimonios nativos directos sobre la percepción que estas comunidades tienen de sí mismas, es sumamente difícil indicar e identificar etnicidades con su correspondiente cultura material. ${ }^{20}$ Así pues, y considerando la presente discusión sobre organización y práctica políticas, podríamos avanzar la hipótesis de que la etnicidad en el Levante - una vez más, especialmente en el Levante meridional durante la Edad del Hierro- podría haber estado íntimamente ligada a las realidades políticas que el patronazgo territorial ejercido por los pequeños reyes locales imponía sobre los sujetos que habitaban sus respectivas comunidades políticas (cf. Benz, 2016: 109; Niesiołowski-Spanò, 2016b). En este sentido, podríamos proponer, pues, que lo que hace particularmente "israelita" a un habitante del territorio por el que se extiende el reino de Israel, lo que hace particularmente "moabita" a un habitante del territorio por el que se extiende el reino de Moab, etc., es esencialmente su subordinación personal y política al monarca (o a alguno de sus subalternos) de estas comunidades políticas. Así pues, sería oportuno explorar en el futuro esta noción de "etnicidad clientelar", de acuerdo con la cual la identidad étnica de una población se encuentra determinada no solamente por factores de identificación primaria como el parentesco, la filiación genealógica, el territorio habitado y la oposición a la alteridad de otra población vecina, sino también por una actitud flexible, adaptativa y situacional hacia situaciones políticas cambiantes en el ámbito regional. Desde esta perspectiva, la creación de una identidad étnica se ampara entonces en la articulación de redes de subordinación y pertenencias políticas. $^{21}$ 


\section{Conclusión}

Entre 1300 y 900 a.n.e. aproximadamente, la amplia región de Siria-Palestina experimentó una serie de disrupciones y re-estructuraciones, notablemente en la escena política de todo su espacio territorial. El consenso historiográfico predominante hasta tiempos recientes ha dictaminado que un cambio radical en las estructuras sociopolíticas tuvo lugar, marcando una transición de un predominio de comunidades políticas ("estados") territoriales en el Bronce Tardío a otro de comunidades políticas étnicas o "nacionales" durante el Hierro II. La discusión concisa que hemos ofrecido aquí intentó desafiar esta comprensión y observar, en cambio, las permanencias fundamentales que atravesaron y resurgieron luego de la crisis del siglo XII, como las relaciones de tipo patrón-cliente, no sin modificaciones, por supuesto, pero manteniéndose como vehículo conceptual y también de praxis para expresar subordinación política personal. En este sentido, es menester realizar a futuro una reinterpretación más profunda de las características y las dinámicas de los llamados "estados étnicos/nacionales" del Hierro II a la luz de la permanencia de estructuras y prácticas sociopolíticas que pueden observarse -si se dispone uno a hacerlo a través de la información y la mirada conceptual que ofrece el registro etnográfico- en la información epigráfico-textual y arqueológica. Esta reinterpretación abre también la posibilidad de considerar a la subordinación política como un factor clave en la construcción de la identidad étnica de los habitantes de los reinos del Levante durante la Edad del Hierro. 
》Akkermans, P. P. M. y Schwartz, G. M. (2003). The Archaeology of Syria: From Complex Hunter-Gatherers to Early Urban Societies (c. 16,000-300 BC). Cambridge: Cambridge University Press.

»Albright, W. F. (1939). The Israelite Conquest of Canaan in the Light of Archaeology, en: Bulletin of the American Schools of Oriental Research 74: 11-23.

» Alt, A. (1959a). Herren und Herrensitze Palästinas im Anfang des zweiten Jahrtausend v. Chr. [1941], en: Alt, A., Kleine Schriften zur Geschichte des Volkes Israel, (ed. por M. Noth). Munich: Beck, III, 57-71.

»Alt, A. (1959b). Die syrische Staatenwelt vor dem Einbruch der Assyrer [1934], en: Alt, A., Kleine Schriften zur Geschichte des Volkes Israel, (ed. por M. Noth). Munich: Beck, III, 214-232.

"Bagg, A. M. (2013). Palestine under Assyrian Rule: A New Look at the Imperial Policy in the West, en: Journal of the American Oriental Society 133: 119-144.

»Barth, F. (1969). Pathan Identity and Its Maintenance, en: Barth, F. (ed.), Ethnic Groups and Boundaries: The Social Organization of Culture Difference. Boston (MA): Little, Brown \& Co, 117-134.

» Barth, F. (1981). Ethnic Processes on the Pathan-Baluch Boundary, en: Barth, F., Features of Person and Society in Swat. Selected Essays of Fredrik Barth, Vol. II. Londres: Kegan \& Paul, 93-102.

» Baruchi-Unna, A. (2018). 'Your Servant and Son I Am': Aspects of the Assyrian Imperial Experience of Judah, en: Aster, S. Z. y Faust, A. (eds.), The Southern Levant under Assyrian Domination. University Park (PA): Eisenbrauns, 119-138.

»Belmonte, J. A. (2003). Cuatro estudios sobre los dominios territoriales de las ciudades-estado fenicias, (Cuadernos de Arqueología Mediterránea, 9). Barcelona: AUSA.

"Benz, B. C. (2016). The Land before the Kingdom of Israel: A History of the Southern Levant and the People who Populated It, (HACL, 7). Winona Lake (IN): Eisenbrauns.

»Berlejung, A. (2012). The Assyrians in the West: Assyrianization, Colonialism, Indifference, or Development Policy?, en: Nissinen, M. (ed.), IOSOT Congress Volume Helsinki 2010, (VTSup, 148). Leiden: E.J. Brill, 21-60.

"Berman, B. J. (1998). Ethnicity, Patronage and the African State: The Politics of Uncivil Nationalism, en: African Affairs 97: 305-341.

"Berman, B. J., Cottrell, J. y Ghai, Y. (2009). Patrons, Clients, and Constitutions: Ethnic Politics and Political Reform in Kenya, en: Canadian Journal of African Studies 43: 462-506.

»Buccellati, G. (1967). Cities and Nations of Ancient Syria: An Essay on Political Institutions with Special Reference to the Israelite Kingdoms, (Studi Semitici, 92). Roma: Università degli Studi di Roma "La Sapienza».

»Buccellati, G. (2013). Alle origini della politica. La formazione e crescita dello stato in Siria-Mesopotamia. Milán: Jaca Books.

» Bunimovitz, Sh. (1994). The Problem of Human Resources in Late Bronze Age 
Palestine and Its Socioeconomic Implications, en: Ugarit Forschungen 26: 1-20.

"Cline, E. H. (2014). 1177 B.C.: The Year Civilization Collapsed, (Turning Points in Ancient History). Princeton: Princeton University Press.

»Coote, R. B. y Whitelam, K. W. (1987). The Emergence of Early Israel in Historical Perspective, (SWABAS, 5). Sheffield: Almond Press.

"Cooper, L. (2010). States of Hegemony: Early Forms of Political Control in Syria during the Third Millennium BC, en: Bolger, D. y Maguire, L. C. (eds.), Development of Pre-State Communities in the Ancient Near East. Oxford: Oxbow Books, 87-94.

»Dever, W. G. (1994). From Tribe to Nation: State Formation Processes in Ancient Israel, en: Mazzoni, S. (ed.), Nuove fondazioni nel Vicino Oriente antico: realtà e ideologia. Pisa: Giardini, 213-229.

"Dever, W. G. (1997). Archaeology, Urbanism and the Rise of the Israelite State, en: Aufrecht, W. E., Mirau, N. A. y Gauley, S. W. (eds.), Urbanism in Antiquity: From Mesopotamia to Crete, (JSOTSup, 244). Sheffield: Sheffield Academic Press, 172-193.

»Dever, W. G. (2007). Ethnicity and the Archaeological Record: The Case of Early Israel, en: Edwards, D. R. y Thomas McCollough, C. (eds.), The Archaeology of Difference: Gender, Ethnicity, Class and the "Other" in Antiquity. Studies in Honor of Eric M. Meyers, (AASOR, 60/61). Boston: ASOR, 49-66.

» Dornemann, R. H. (2003). State Formation in Syria at the Beginning of the Iron Age, en: Clark, D. R. y Matthews, V. H. (eds.), One Hundred Years of American Archaeology in the Middle East: Proceedings of the American Schools of Oriental Research Centennial Celebration, Washington, DC April 2000. Boston: ASOR, 199214 .

» Fales, F.M. (2017). Ethnicity in the Assyrian Empire: A View from the Nisbe, [III] Arameans and Related Tribalists, en: Heffron, Y., Stone, A. y Worthington, M. (eds.), At the Dawn of History: Ancient Near Eastern Studies in Honour of J. N. Postgate. Vol. 1. Winona Lake (IN): Eisenbrauns, 133-177.

" Faust, A. (2012). The Archaeology of Israelite Society in Iron Age II. Winona Lake (IN): Eisenbrauns.

" Finkelstein, I. (1988). The Archaeology of the Israelite Settlement. Jerusalén: Israel Exploration Society.

»Finkelstein, I. (1994). The Emergence of Israel: A Phase in the Cyclic History of Canaan in the Third and Second Millennia BCE, en: Finkelstein, I. y Na'aman, N. (eds.), From Nomadism to Monarchy: Archaeological and Historical Aspects of Early Israel. Jerusalén: Israel Exploration Society, 150-178.

»Finkelstein, I. (2003). City-States to States: Polity Dynamics in the 1oth-9th Centuries B.C.E., en: Dever, W. G. y Gitin, S. (eds.), Symbiosis, Symbolism, and the Power of the Past: Canaan, Ancient Israel, and Their Neighbors from the Late Bronze Age through Roman Palaestina. Winona Lake (IN): Eisenbrauns, 75-83.

» Finkelstein, I. (2010). A Great United Monarchy? Archaeological and Historical Perspectives, en: Kratz, R. G. y Spieckermann, H. (eds.), One God - One Cult One Nation: Archaeological and Biblical Perspectives, (BZAW, 405). Berlín: W. de Gruyter, 3-28.

"Finkelstein, I. (2013). The Forgotten Kingdom: The Archaeology and History of Northern Israel, (ANEM, 5). Atlanta: Society of Biblical Literature. 
» Finkelstein, I. y Na'aman, N. (eds.). (1994). From Nomadism to Monarchy: Archaeological and Historical Aspects of Early Israel. Jerusalén: Israel Exploration Society.

»Finkelstein, I. y Na'aman, N. (2005). Shechem of the Amarna Period and the Rise of the Northern Kingdom of Israel, en: Israel Exploration Journal 55: 172-193.

» Fritzsche, P. (2004). Stranded in the Present: Modern Time and the Melancholy of History. Cambridge (MA): Harvard University Press.

» Gaddis, J. L. (2002). The Landscape of History: How Historians Map the Past. Oxford: Oxford University Press.

"Garfinkel, Y. et al. (2012). State Formation in Judah: Biblical Tradition, Modern Historical Theories, and Radiometric Dates at Khirbet Qeiyafa, en: Radiocarbon 54: 359-369.

" Geary, P.J. (2002). The Myth of Nations: The Medieval Origins of Europe. Princeton: Princeton University Press.

» Gitin, S., Mazar, A. y Stern, E. (eds.). (1998). Mediterranean Peoples in Transition: Thirteenth to Early Tenth Century BCE. Jerusalén: Israel Exploration Society.

» Grabbe, L. L. (ed.). (2008). Israel in Transition: From the Late Bronze II to Iron Ila (ca. 1250-850 B.C.E.). Volume 1: The Archaeology, (LHBOTS, 491 / ESHM, 7). Londres: T \& T Clark.

" Grabbe, L. L. (ed.). (2010). Israel in Transition 2: From the Late Bronze II to Iron Ila (ca. 1250-850 B.C.E.). Volume 2: The Texts, (LHBOTS, 521 / ESHM, 8). Londres: T \& T Clark.

»Hallo, W. W. (ed.). (2003). The Context of Scripture. Vol. 2: Monumental Inscriptions from the Biblical World. Leiden: E.J. Brill.

»Holladay, J. S. Jr. (1995). The Kingdoms of Israel and Judah: Political and Economic Centralization in the Iron II A-B, en: Levy, T. E. (ed.), The Archaeology of Society in the Holy Land. Nueva York: Facts on File, 368-398.

» Jasmin, M. (2006a). The Political Organization of the City-States in Southwestern Palestine in the Late Bronze Age IIB (13th Century BC), en: Maeir, A. M. y de Miroschedji, P. (eds.), 'I Will Speak the Riddles of Ancient Times': Archaeological and Historical Studies in Honor of Amihai Mazar on the Occasion of his Sixtieth Birthday. Winona Lake (IN): Eisenbrauns, 161-191.

» Jasmin, M. (2006b). L'étude de la transition du Bronze recent Il au Fer I en Palestine méridionale, (BAR International Series, 1495). Oxford: BAR.

» Joffe, A. H. (2002). The Rise of Secondary States in the Iron Age Levant, en: Journal of the Economic and Social History of the Orient 45: 425-467.

" Jones, S. (1997). The Archaeology of Ethnicity: Constructing Identities in the Past and Present. Londres: Routledge.

»Kelle, B. E. (2002). What's in a Name? Neo-Assyrian Designations for the Northern Kingdom and Their Implications for Israelite History and Biblical Interpretation, en: Journal of Biblical Literature 121: 639-666.

" Killebrew, A. E. (2005). Biblical Peoples and Ethnicity: An Archaeological Study of Egyptians, Canaanites, Philistines, and Early Israel, (ABS, 9). Atlanta: Society of Biblical Literature.

»Killebrew, A. E. y Lehmann, G. (eds.). (2013). The Philistines and Other "Sea Peoples" in Text and Archaeology, (ABS, 15). Atlanta: Society of Biblical Literature. 
» Kleiman, A. (2016). The Damascene Subjugation of the Southern Levant as a Gradual Process (ca. 842-80o BCE), en: Sergi, O., Oeming, M. y de Hulster, I. J. (eds.), In Search for Aram and Israel: Politics, Culture and Identity, (ORA, 20). Tubinga: Mohr Siebeck, 57-76.

》 Kletter, R. (2004). Chronology and United Monarchy: A Methodological Review, en: Zeitschrift des Deutschen Palästina-Vereins 120: 13-54.

" Kletter, R. (2014). In the Footsteps of Bagira: Ethnicity, Archaeology, and 'Iron Age I Ethnic Israel', en: Approaching Religion 4: 2-15.

»Knauf, E. A. (1992). The Cultural Impact of Secondary State Formation: The Cases of the Edomites and Moabites, en: Bienkowski, P. (ed.), Early Edom and Moab: The Beginning of the Iron Age in Southern Jordan, (SAM, 7). Sheffield: J.R. Collis, 47-54.

»Knauf, E. A. y Guillaume, P. (2016). A History of Biblical Israel: The Fate of the Tribes and Kingdoms from Merenptah to Bar Kochba, (WANEM). Sheffield: Equinox.

»Koch, I. (2018). The Egyptian-Canaanite Interface as Colonial Encounter: A View from Southwest Canaan, en: Journal of Ancient Egyptian Interconnections 18: 2439.

»LaBianca, Ø. y Younker, R. W. (1995). The Kingdoms of Ammon, Moab and Edom: The Archaeology of Society in Late Bronze/Iron Age Transjordan (ca. 1400-500 BCE), en: Levy, T. E. (ed.), The Archaeology of Society in the Holy Land. Nueva York: Facts on File, 399-415.

»Lehmann, G. y Niemann, H. M. (2006). Klanstruktur und charismatische Herrschaft: Juda und Jerusalem 1200-900 v. Chr., en: Theologische Quartalschrift 186: 134-159.

"Lemarchand, R. (1972). Political Clientelism and Ethnicity in Tropical Africa: Competing Solidarities in Nation-Building, en: The American Political Science Review 66: 68-90.

"Lemche, N. P. (1985). Early Israel: Anthropological and Historical Studies on the Israelite Society before the Monarchy, (VTSup, 37). Leiden: E. J. Brill.

"Lemche, N. P. (2002). Chronology and Archives-When Does the History of Israel and Judah Begin?, en: Gunn, D. M. y McNutt, P. M. (eds.), 'Imagining' Biblical Worlds: Studies in Spatial, Social and Historical Constructs in Honor of James W. Flanagan, (JSOTSup, 359). Sheffield: Sheffield Academic Press, 264-276.

"Leonard-Fleckman, M. (2018). The bìt X Formula in Assyrian Documentation and Aramaean Social Structure, en: Hebrew Bible and Ancient Israel 7: 140-171.

» Lipschits, O. (2004). Ammon in Transition from Vassal Kingdom to Babylonian Province, en: Bulletin of the American School of Oriental Research 335: 37-52.

" Liverani, M. (1997). Ramesside Egypt in a Changing World: An Institutional Approach, en: Colonna, G. (ed.), L'impero ramesside. Convegno internazionale in onore di Sergio Donadoni, (Vicino Oriente Quaderno, 1). Roma: Università degli Studi di Roma "La Sapienza», 101-115.

"Liverani, M. (2002). Stati etnici e città-stato: una tipologia storica per la prima età del ferro, en: Molinos, M. y Zifferero, A. (eds.), Primi popoli d'Europa. Proposte e riflessioni sulle origini della civiltà nell'Europa mediterranea. Florencia: All'Insegna del Giglio, 33-47.

» Liverani, M. (2014). The Ancient Near East: History, Society, Economy. Londres: Routledge. 
»London, G. (1989). A Comparison of Two Contemporary Lifestyles of the Late Second Millennium B.C.E., en: Bulletin of the American Schools of Oriental Research 273: 37-55.

》Masetti-Rouault, M. G. (2009). Cultures in Contact in the Syrian Lower Middle Euphrates Valley: Aspects of the Local Cult in the Iron Age II, en: Syria 86: 141147.

"Masetti-Rouault, M. G. (en prensa). The View from Aram: Aramaic States in Northern Syria and the Expansion of the Assyrian Empire, en: Margueron, J.-C., de Miroschedji, P. y Thalmann, J. P. (eds.), Proceedings of the 3 rd ICAANE (Paris, 15-19 Avril 2002). Winona Lake (IN): Eisenbrauns.

»Megill, A. (2007). Historical Knowledge, Historical Error: A Contemporary Guide to Practice. Chicago: The University of Chicago Press.

» Morris, E. (2018). Ancient Egyptian Imperialism. Chichester: Wiley Blackwell.

»Na'aman, N. (2007). The Northern Kingdom in the Late Tenth-Ninth Centuries BCE, en: Williamson, H. G. M. (ed.), Understanding the History of Ancient Israel, (Proceedings of the British Academy, 143). Oxford: The British Academy, 399-418.

"Nam, R. S. (2012). Power Relations in the Samaria Ostraca, en: Palestine Exploration Quarterly 144: 155-163.

» Niemann, H. M. (2008). A New Look at the Samaria Ostraca: The King-Clan Relationship, en: Tel Aviv 35: 249-266.

"Niemeyer, H. G. (2000). The Early Phoenician City-States on the Mediterranean: Archaeological Elements for their Description, en: Hansen, M. H. (ed.), A Comparative Study of Thirty City-State Cultures, (Historisk-filosofiske Skrifter 21 / Det Kongelige Danske Videnskabernes Selskab). Copenhague: C. A. Reitzels Forlag, 89-115.

» Niesiołowski-Spanò, Ł. (2016a). Goliath's Legacy: Philistines and Hebrews in Biblical Times, (Philippika: Altertumswissenschaftliche Abhandlungen, 83). Wiesbaden: Harrassowitz.

»Niesiołowski-Spanò, Ł. (2016b). Functional Ethnicity: Or, How to Describe the Societies of Ancient Palestine, en: Ugarit Forschungen 47: 191-203.

»Okamura, J. Y. (1981). Situational Ethnicity, en: Ethnic and Racial Studies 4: 452465 .

»Oren, E. D. (1984). 'Governors' Residences' in Canaan during the New Kingdom: A Case Study of Egyptian Administration, en: Journal of the Society for the Study of Egyptian Antiquity 14: 37-56.

"Osborne, J. F. (2014). Monuments and Monumentality, en: Osborne, J. F. (ed.), Approaching Monumentality in Archaeology. Albany: State University of New York Press, 1-19.

"Pfoh, E. (2008). Dealing with Tribes and States in Ancient Palestine: A Critique on State Formation Theories in the Archaeology of Israel, en: Scandinavian Journal of the Old Testament 22: 86-113.

»Pfoh, E. (2013). Loyal Servants of the King: A Political Anthropology of Subordination in Syria-Palestine (ca. 1600-60o BCE), en: Palamedes: A Journal of Ancient History 8: 25-41.

"Pfoh, E. (2016). Syria-Palestine in the Late Bronze Age: An Anthropology of Politics and Power, (CIS). Londres: Routledge. 
»Pfoh, E. (2018). Socio-Political Changes and Continuities in the Levant (1300900 BCE), en: Niesiołowski-Spanò, Ł. y Węcowski, M. (eds.), Change, Continuity, and Connectivity: North-Eastern Mediterranean at the Turn of the Bronze Age and in the Early Iron Age, (Philippika - Altertumskundliche Abhandlungen, 118). Wiesbaden: Harrassowitz, 57-67.

»Pfoh, E. (2019). Prestige and Authority in the Southern Levant during the Amarna Age, en: Maeir, A. M., Shai, I. y McKinny, C. (eds.), The Late Bronze and Early Iron Ages of Southern Canaan, (ABW, 2). Berlín: W. de Gruyter, 247-261.

»Pfoh, E. (en prensa). Feudalism and Vassalage in Twentieth-Century Assyriology, en: Garcia-Ventura, A. y Verderame, L. (eds.), Perspectives on the History of Ancient Near Eastern Studies. University Park (PA): Eisenbrauns / PSU Press.

» Porzia, F. (2018). 'Imagine There's No Peoples'. A Claim against the Identity Approach in Phoenician Studies through Comparison with the Israelite Field, en: Rivista di Studi Fenici XLVI: 11-27.

"Rainey, A. F. (2015). The El-Amarna Correspondence: A New Edition of the Cuneiform Letters from the Site of El-Amarna based on Collations of all Extant Tablets, 2 vols. (ed. por Schniedewind, W. y Cochavi-Rainey, Z.; HdO, 110). Leiden: E. J. Brill.

》 Routledge, B. (2004). Moab in the Iron Age: Hegemony, Polity, Archaeology, (Archaeology, Culture, and Society). Filadelfia: University of Pennsylvania Press.

" Routledge, B. (2017). Is There an Iron Age Levant?, en: Revista del Instituto de Historia Antigua Oriental ns 18: 49-76.

»Sader, H. (2016). The Formation and Decline of the Aramaean States in Iron Age Syria, en: Kessler, R., Sommerfeld, W. y Tramontini, L. (eds.), State Formation and State Decline in the Near and Middle East. Wiesbaden: Harrassowitz, 61-76.

»Savage, S. H. y Falconer, S. E. (2003). Spatial and Statistical Inference of Late Bronze Age Polities in the Southern Levant, en: Bulletin of the American Schools of Oriental Research 330: 31-45.

» Schäfer-Lichtenberger, Ch. (1996). Sociological and Biblical Views on the Early State, en: Fritz, V. y Davies, P. R. (eds.), The Origins of the Ancient Israelite States, (JSOTSup, 228). Sheffield: Sheffield Academic Press, 78-105.

»Schloen, J. D. (2016). Economy and Society in Iron Age Israel and Judah: An Archaeological Perspective, en: Niditch, S. (ed.), The Wiley Blackwell Companion to Ancient Israel. Oxford: Blackwell, 433-453.

"Sergi, O. (2015). State Formation, Religion and 'Collective Identity' in the Southern Levant, en: Hebrew Bible and Ancient Israel 4: 56-77.

"Silberman, N. A. (1998). The Sea Peoples, the Victorians, and Us: Modern Social Ideology and Changing Archaeological Interpretations of the Late Bronze Age Collapse, en: Gitin, S., Mazar, A. y Stern, E. (eds.), Mediterranean Peoples in Transition: Thirteenth to Early Tenth Centuries BCE. Jerusalén: Israel Exploration Society, 268-275.

» Skjeggestad, M. (1992). Ethnic Groups in Early Iron Age Palestine: Some Remarks on the Use of the Term 'Israelite' in Recent Research, en: Scandinavian Journal of the Old Testament 6: 159-186.

»Smith, A. T. (2003). The Political Landscape: Constellations of Authority in Early Complex Polities. Berkeley: University of California Press.

"Southall, A. (1988). The Segmentary State in Africa and Asia, en: Comparative Studies in Society and History 30: 52-82. 
» Strange, J. (2000). The Philistine City-States, en: Hansen, M. H. (ed.), A Comparative Study of Thirty City-State Cultures, (Historisk-filosofiske Skrifter, 21 / Det Kongelige Danske Videnskabernes Selskab). Copenhague: C. A. Reitzels Forlag, 129-139.

»Tebes, J. M. (2016). The Kingdom of Edom? A Critical Reappraisal of the Edomite State Model, en: Milevski, I. y Levy, T. E. (eds.), Framing Archaeology in the Near East: The Application of Social Theory to Fieldwork (New Directions in Anthropological Archaeology). Sheffield: Equinox, 113-122.

» Thompson, T. L. (1992). Early History of the Israelite People: From the Written and Archaeological Sources, (SHANE, 4). Leiden: E. J. Brill.

» Tibi, B. (1990). The Simultaneity of the Unsimultaneous: Old Tribes and Imposed Nation-States in the Modern Middle East, en: Khoury, P. S. y Kostiner, J. (eds.), Tribes and State Formation in the Middle East. Berkeley: University of California Press, 127-152.

"Vera Chamaza, G. W. (2005). Die Rolle Moabs in der neuassyrischen Expansionspolitik, (AOAT, 321). Münster: Ugarit-Verlag.

"Ward, W. A. y Joukowsky, M. (eds.). (1992). The Crisis Years: The 12th Century B.C.: From Beyond the Danube to the Tigris. Dubuque: Kendall/Hunt.

"Whitelam, K. W. (2002). Palestine during the Iron Age, en: Barton, J. (ed.), The Biblical World. Vol. 1. Londres: Routledge, 386-410.

»Wiedemann, F., Hofmann, K. P. y Gehrke, H.-J. (eds.). (2017). Vom Wandern der Völker: Migrationserzählungen in den Altertumwissenschaften, (BSAW, 41). Berlín: Edition Topoi.

"Yasur-Landau, A. (2010). The Philistines and Aegean Migration at the End of the Late Bronze Age. Cambridge: Cambridge University Press.

» Zwingenberger, U. (2001). Dorfkultur der frühen Eisenzeit in Mittelpalästina, (OBO, 180). Gotinga: Vandenhoeck \& Ruprecht. 